\title{
Free choice of signaled vs unsignaled scrambled electric shock with rats
}

\author{
MARK S. CRABTREE and BRIAN M. KRUGER \\ Wright State University, Dayton, Ohio 45431
}

\begin{abstract}
After forced exposure to both sides of a shuttlebox, 15 rats were given a choice between signaled scrambled electric shock in one half and unsignaled scrambled shock in the other. The interstimulus intervals between tone and shock were $2,4,8,16$, or 32 sec. Signal offset contingencies were: off 1 sec before shock, off at shock onset, or off at shock offset. The main finding of no preference between signaled and nonsignaled shock supports the view that signaled shock will be preferred only when it is modifiable.
\end{abstract}

Lockard (1963) reported that rats prefer signaled unscrambled shock to unsignaled unscrambled shock. One potential explanation for this preference for signaled unscrambled shock is that a rat may contact only grids of the same polarity and thus avoid the shock. While Lockard (1963) found that complete avoidance was not a function of signaling, she noted that certain postures could reduce the noxiousness of shock (Campbell \& Teghtsoonian, 1958). The use of grid scramblers should reduce complete avoidances and minimize the modification of perceived shock through postural adjustments on the grids. However, the rat can still avoid shock in some apparatuses by standing on a single grid and leaning against or holding onto a nonelectrified surface such as a wall or ceiling. Such problems (Furedy \& Walters, Note 1) can be reduced by a fixed electrode method of administration where physical modification of shock can be minimized or perhaps eliminated. Biederman and Furedy (1973) found no preference for signaled shock in a forced-choice situation with either tailshock or scrambled shock administered through foot grids. The present report presents the findings for scrambled shock in a two-compartment free-choice apparatus in which vertical grids (walls) and doorways were also electrified.

\section{METHOD}

\section{Subjects and Design}

The 3 by 5 experimental design had the following factors: the interstimulus interval or time from tone onset to shock onset $(2,4$, 8,16 , or $32 \mathrm{sec}$ ) and the tone offset contingency ( $1 \mathrm{sec}$ before shock onset, simultaneous with shock onset, or simultaneous with shock offset, designated as before, on, and off conditions, respectively). Fifteen naive male Long-Evans hooded rats, ranging in weight from 193 to $334 \mathrm{~g}$, were assigned randomly to the 15 conditions.

\section{Apparatus}

The translucent white plastic shuttlebox, illuminated at $18.3 \mathrm{~lx}$

This article is sponsored by P. E. Campbell, who takes full editorial responsibility for its contents. Reprints may be obtained from the second author, Department of Psychology, Wright State University, Dayton, Ohio 45431. and located in a sound-reduction room with $67-\mathrm{dB}$ white-noise background, was maintained at $276^{\circ} \mathrm{K}$. A $1,000-\mathrm{Hz}$ tone at $77 \mathrm{~dB}$ was employed as the signal. On the nonsignal side, a $1.91-\mathrm{cm}$-wide black stripe was centered lengthwise on the white subfloor, $2.54 \mathrm{~cm}$ below the $2.38-\mathrm{mm}$-diam floor grids. Floor grids were spaced every $1.27 \mathrm{~cm}$, and the vertical grids covering the walls of each $8.08 \mathrm{x}$ $28.40 \times 20.32 \mathrm{~cm}$ chamber were spaced every $6.35 \mathrm{~mm}$. The chambers were separated by an aluminum plate for forced enclosures. For choice sessions, a plate with a $7.62 \times 12.07 \mathrm{~cm}$ doorway was employed; there was no hurdle between chambers. Two horizontal infrared beams located $3.18 \mathrm{~cm}$ above the floor and 2.54 and $7.62 \mathrm{~cm}$ inside the doorway of each chamber were used in sensing the rat's location.

The 2-sec-duration shocks were delivered by an I-O-Tek Model 101 shock scrambler. The momentary potentials were ground for one output and $+110 \mathrm{~V}$ dc via $47 \mathrm{~K}$ resistors for the other eight outputs. All vertical grids and plates were connected to one output, and the other eight outputs were connected to very eighth floor grid. Polarity changes occurred sequentially at the rate of 120 changes/sec.

\section{Procedure}

A 3 -h pretest session with only tone presentations was given to each rat. The purpose of the pretest session was to establish a baseline preference for either side of the shuttlebox. The tone was presented on only one side of the shuttlebox throughout the entire experiment. A 12-h acquisition session (divided into four 3-h subsessions) was followed by a 6 -h extinction session (divided into two 3-h subsessions). During the extinction session, as during the pretest session, shock was withheld and only the tone presented. Interpresentation intervals, varying from 60 to $180 \mathrm{sec}$ in 20 -sec intervals, were block randomized. Each rat was forcibly exposed to (enclosed in) each side of the shuttlebox for a $1 / 2 \mathrm{~h}$ per side prior to the pretest session (tone on one side) and again prior to the acquisition session (tone paired with shock on one side).

The number of secorids on the signal side were counted for each 3-h subsession. (A rat mostly on the signal side was considered on the signal side as long as the first beam inside the nonsignal side was not obstructed.) The number of crossovers per subsession were also counted. A crossover was defined as beginning when only beams in one side were occluded and as ending when only beams in the other side were occluded. The decision to present the warning signal required that at the scheduled signal onset the rat be on the signal side. A rat's breaking a beam on the nonsignal side during signal presentation did not result in signal termination.

The number of seconds on the signal side for each subsession were linearly transformed to percentages. To permit comparison of acquisition and extinction measures, average percentages and crossovers for acquisition and extinction subsessions were calculated. Adjusted measures were obtained by subtracting pretest measures. 


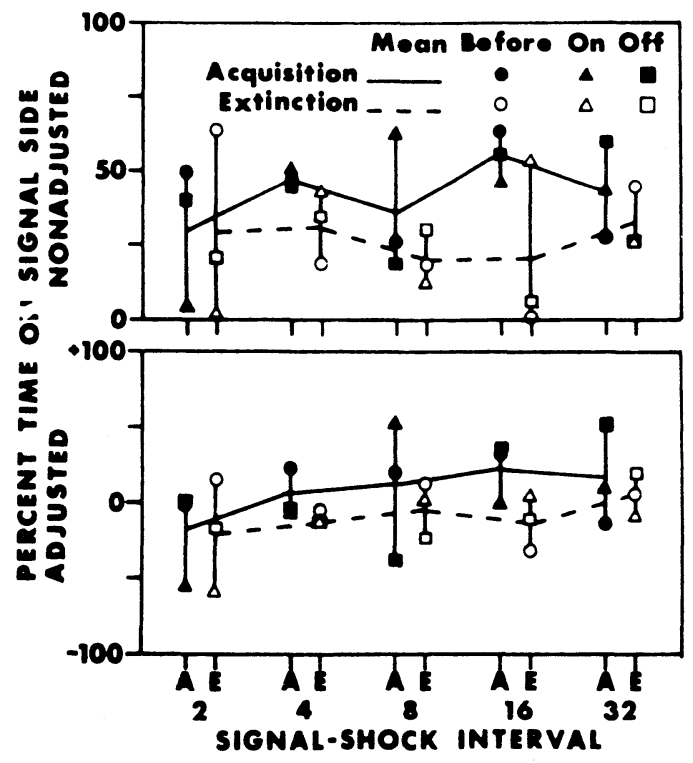

Figure 1. Adjusted and nonadjusted percentage of time on signal side during acquisition and extinction for each subject.

A three-way analysis of variance for a design with one repeated measures factor and one observation per cell was employed for the analysis of nonadjusted and adjusted measures. The appropriateness of the model was assessed with tests for nonadditivity after Tukẹ (1949).

\section{RESULTS}

Tests for nonadditivity failed to reject the hy pothesis of addivity, $\mathrm{p}>.15$ for all tests. The model was accepted as appropriate for all analyses.

There were no significant main effects or

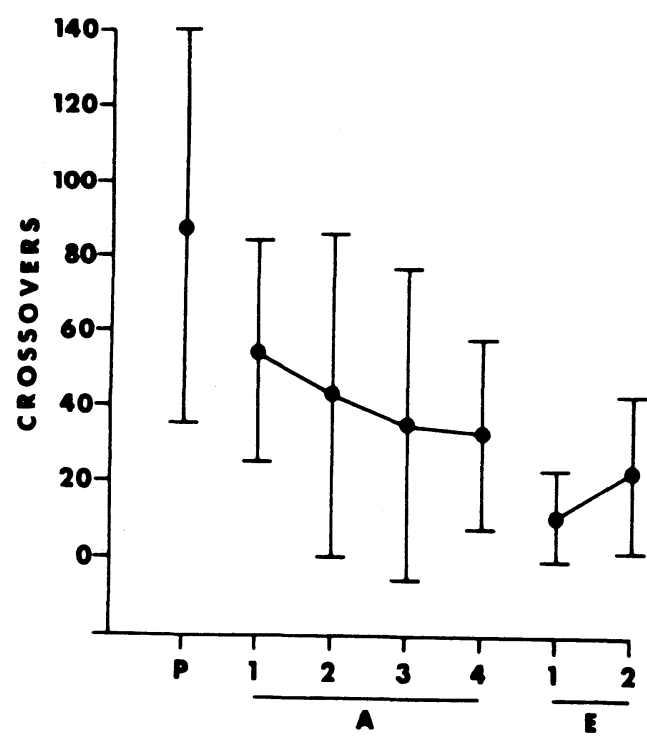

Figure 2. Mean and standard deviation of the number of crossovers for all subjects as a function of pretest, acquisition, and extinction sessions. interactions for nonadjusted or adjusted percent time on signal side. The nonadjusted and adjusted percentages of time on the signal side during acquisition and extinction are shown in Figure 1. A nonsignificant proportion of subjects ( 8 of 15) spent more time on the signal side during acquisition than during the pretest.

The means and standard deviations for all subjects for nonadjusted crossover measures are shown in Figure 2. There was a significant decrease across subsessions in the nonadjusted number of crossovers in acquisition, $F(3,24)=4.49 . p<.05$. The average number of crossovers per subsession significantly decreased from acquisition to extinction for both the nonadjusted and the adjusted measures, $F(1,8)=$ 8.65. $\mathrm{p}<.05$, and $\mathrm{F}(1.8)=20.27, \mathrm{p}<.01$, respectively. And there was a significant increase in the number of crossovers across subsessions in extinction for both nonadjusted and adjusted measures. $F(1,8)=8.87, p<.05$, and $F(1,8)=8.92$, $\mathrm{p}<.05$. respectively.

\section{DISCUSSION}

The signiticant decrease during acquisition and increase during extinction in number of crossovers suggests that session length was adequate for conditioning of aversive properties. The increase during extinction seems to rule out the possibility that the decrease during acquisition was due exclusively to fatigue or habituation to the experimental situation. When clearly modifiable unscrambled shock has been employed in free-choice situations. preferences have been evident after $6 \mathrm{~h}$ or less (e.g.. Lockard, 1963; Furedy \& Walters. Note 1), or about half the acquisition time of the present study.

Since the average percent time on the signal side was greater during acquisition than during the pretest for only 8 of 15 rats (and less for the other 7 ), and since there were no significant effects of the acquisition subsessions factor on the percentage of time spent on the signal side. it may be concluded that there was no demonstrated preference for either signaled or unsignaled shock. Thus. this study supports the findings of other studies which have employed scrambled shock and found no preference (e.g. Biederman \& Furedy. 1973) and seems to support the view that signaled shock is preferred only when that information permits preparation for and modification of shock (Perkins. 1955). The results do not support the view that reduction in arousal produced by uncertainty concerning time of occurrence is sufficient to produce a preference for signaled shock regardless of whether the noxious event is modifiable (Berlyne. 1960. chap. 7).

\section{REFERENCE NOTE}

1. Furedy. J. J.. \& Walters. G. C. Preference for signaled. supposedly-unmoditiable shock as a function of scrambling the grid. Paper presented at the meeting of the Psychonomic Society in St. Louis. November 1970.

\section{REFERENCES}

Berlyne. D. E. Conflict, arousal. and curiosity. New York: McGraw-Hill. 1960.

Biederman. G. E.. \& Furedy. J. J. Preference-for-signaled-shock phenomenon: Effects of shock modifiability and light reinforcement. Journal of Experimental Psychology. 1973. 100. 380-38b. 
Campbell. B. A.. \& Teghtsoonian. R. Electrical and behavioral effects of different types of shock stimuli on the rat. Journal of Comparative and Physiological Psychology. 1958. 51. 185-192.

LOCKARD. J. S. Choice of a warning signal or no warning signal in an unavoidable shock situation. Journal of Comparative and Physiological Psychology. 1963, 56. 526-530.
Perkins. C. C.. JR. The stimulus conditions which follow learned responses. Psychological Review, 1955, 62, 341-348.

TLKEY, J. W. One degree of freedom for non-additivity. Biometrics. 1949. 5. 232-242.

(Received for publication June 21. 1975.) 